\title{
Edge diffraction in sunlight
}

Vincenzo Greco, Giuseppe Molesini, R. Tronconi

Vincenzo Greco, Giuseppe Molesini, R. Tronconi, "Edge diffraction in sunlight," Proc. SPIE 3190, Fifth International Topical Meeting on Education and Training in Optics, (8 December 1997); doi: 10.1117/12.294411 1997, Delft, Netherlands 
Edge diffraction in sunlight

V. Greco, G. Molesini and R. Tronconi

Istituto Nazionale di Ottica, Largo E. Fermi 6, Firenze 50125, Italy

\begin{abstract}
Observations of wave-optics effects in sunlight are reported. In particular, conditions are described that allow for visual detection of diffraction phenomena from line edges. Typical fringe patterns are demonstrated, also showing color features that account for the wavelength dependence of the diffraction process. Hints to optimize the observation are given, outlining the aspects of simplicity and naturalness of the occurrence.

\section{INTRODUCTION}

With laser light, demonstrating wave optics is extremely easy. Experiments on diffraction in particular are reported, also including the use of a computer to collect data, perform the computations and display the results. ${ }^{1,2}$ With natural light such as daylight, several evidences of diffraction are still observed, mostly from grating structures and holes. Spatial coherence effects in sunlight as speckle phenomena have been wonderfully illustrated in a recent note. ${ }^{3}$

Seeing diffraction from a straight edge in sunlight, however, is not very common. Favourable conditions can though be met occasionally; it is then a matter of recognizing the phenomenon and optimizing the observation. Since edge diffraction is likely the simplest and most fundamental case treated by the theory, ${ }^{4}$ it appears worth noting that its experimental evidence is available in everyday life. This paper describes the occurrence of the phenomenon and presents some considerations concerning the educational value of the observation.
\end{abstract}

\title{
2. SEEING THE FRINGES
}

We first noted diffraction fringes on the shadow of a book placed on a desk, while the sunlight was filtered by the rolling shutter of the window early in the morning. A sketch of the observing conditions is drawn in Fig. 1. The narrow aperture of the shutter provides a horizontal slit. The diffracting edge is that of the margin of the page. Light is cast on the desk obliquely, so that the shadow pattern is stretched and the fringes are magnified.

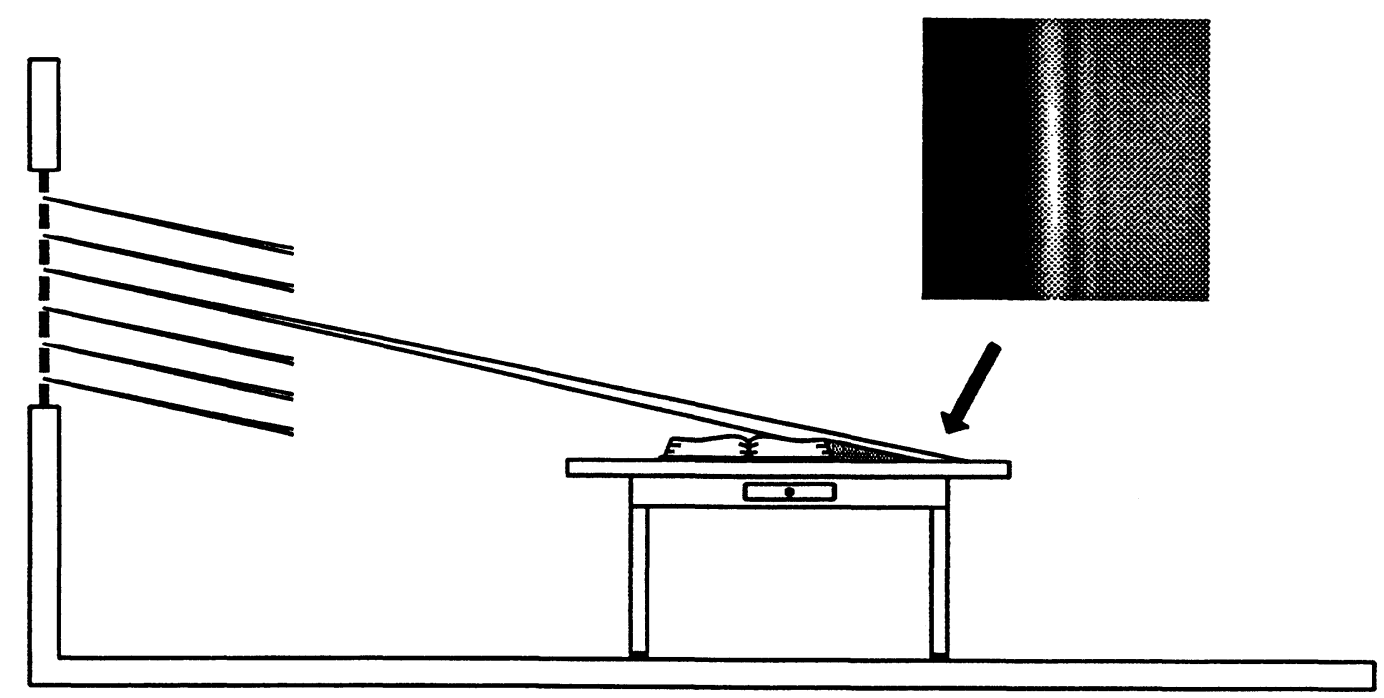

Fig. 1. Case of natural conditions where edge diffraction fringes in sunlight are observed. 
As noted in the cited literature, ${ }^{3}$ sunlight has a spatial coherence width $d$ of approximately $72 \mu \mathrm{m}$. Such an estimate is drawn from the Van Cittert-Zernike theorem by the formula ${ }^{5}$

$$
d=1.22 \lambda / \theta,
$$

where $\lambda$ is the wavelength of the light (assumed $550 \mathrm{~nm}$ in the computations) and $\theta$ is the full angular subtent of the sun $\left(\theta=9.3 \cdot 10^{-3}\right.$ radians, or $\left.0.53^{\circ}\right)$. In the case of a slit the numerical factor is just 1 . For a slit width of $1 \mathrm{~mm}$ at, say, $4 \mathrm{~m}$ distance from the desk the full angular subtent is $0.25 \cdot 10^{-3}$ radians, resulting in a vertical coherence extent of $2.2 \mathrm{~mm}$ (namely, about a factor 30 enhanced). Such a degree of spatial coherence proves to be sufficient for the diffraction pattern to appear; fringes are readily recognized about the shadow border. ${ }^{6}$

Fresnel diffraction at a semi-infinite plane bounded by a sharp straight edge is a classical topics in the optics literature. ${ }^{4,7}$ On a screen nearly normal to the light direction, the diffraction pattern shows a structure made of a smooth shadow about the geometrical projection of the edge, and a series of fringes extending into the bright illuminated area; the pitch of the fringes at farther distances from the edge projection is shorter, and the visibility smaller. As an order of magnitude, the width $w_{0}$ of the first fringe is about

$$
w_{0} \simeq \sqrt{\frac{\lambda z}{2}}
$$

where $z$ is the distance between the diffracting edge and the screen where observation takes place.

The angular resolution $\alpha$ of the human eye may be taken roughly one minute of arc $\left(3 \cdot 10^{-4}\right.$ radians $) .{ }^{8}$ At the nearest point $(250 \mathrm{~mm})$ the minimum resolved pitch $p$ is then $p=0.075 \mathrm{~mm}$. The distance $z_{0}$ where fringes are first seen can be computed by letting

$$
w_{0}\left(z_{0}\right)=p
$$

so that it is obtained

$$
z_{\circ} \simeq \frac{2}{\lambda} p^{2}
$$

At $\lambda=550 \mathrm{~nm}$ as above, the numerical result for $z_{0}$ is about $20 \mathrm{~mm}$. Better observation conditions are achieved at farther distances, of by means of some magnification. In the case of Fig. 1 such magnification is naturally obtained because the plane of observation is tilted. The geometrical magnification factor is $1 / \cos \delta$, being $\delta$ the tilt angle. ${ }^{9}$ The general expression for the width of the first fringe is then

$$
w_{\circ} \simeq \frac{1}{\cos \delta} \sqrt{\frac{\lambda z}{2}} \text {. }
$$

For example, taking $\delta=85^{\circ}$ and $z \simeq 20 \mathrm{~cm}$ the first diffraction fringe extends over a width of nearly $2.7 \mathrm{~mm}$.

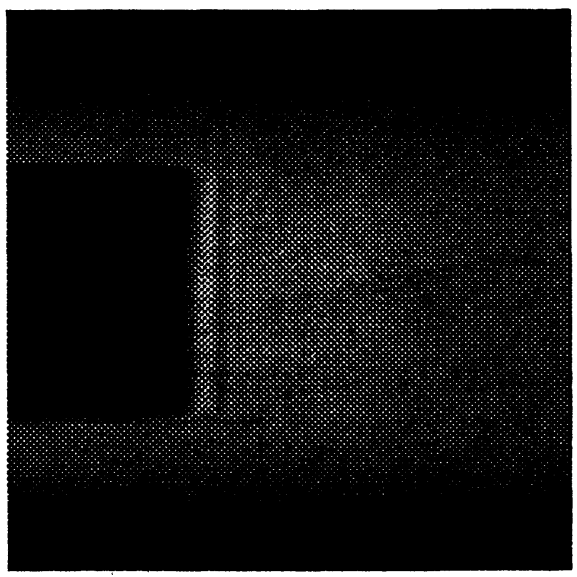

Fig. 2. Diffraction from a sharp edge in sunlight. The shadow belongs to a cube, whose side is $30 \mathrm{~mm}$ long. 
The observation depicted in Fig. 1 was repeated under similar conditions. When the sun was high, use was made of an external mirror to redirect the light against the rolling shutter at a proper angle. In particular we have made records with a tilt angle about $84.3^{\circ}$, resulting in a geometrical magnification factor about 10 . This was sufficient for clearly observing the diffraction fringes and taking pictures. In Fig. 2 a typical pattern is presented; it is produced by a metal cube, placed on the table so that one of its sharp corners is illuminated by sunlight from a window slit. Where the shadow is cast, a white screen is placed for better observation. Although the picture is displayed in black and white, the actual fringes have a color structure, accounting for the wavelength dependence of the diffraction phenomena.

\section{CONCLUDING REMARKS}

There are situations in everyday life where the light displays some spatial coherence effects. This is the case of sunlight penetrating into the room through a chink, or through the slits of a rolling shutter. Peculiar diffraction phenomena then show up; edge diffraction in particular is observed. The diffracting edge should be at some distance from the chink or the slit, and nearly parallel to it. A surface acting as a screen is necessary, where the light is cast: best conditions of observation are met when the surface is illuminated at close grazing incidence, so that the effect is magnified. What appears is a 2-3 fringe system along the edge shadow, with some color feature, typical of diffraction phenomena.

As an experiment for education, the demonstration is very easy, although it requires the right conditions to be observed. Since no laboratory instrument is used, it is useful to point out that diffraction is intrinsic and quite natural. Although the derivation of Eqs. (1), (2) is far from being obvious, the relationships themselves are simple and suitable to making the observations quantitative.

\section{ACKNOWLEDGMENTS}

The authors wish to express their gratitude to S. F. Jacobs for useful comments, discussions and encouragement while making the observations, evaluating the data and presenting the results.

\section{REFERENCES}

1. W. Klein, "The direct display of diffraction patterns using an electric razor", Am. J. Phys. Vol. 54, pp. 956-958 (1986).

2. C. A. Bennett, "A computer-assisted experiment in single-slit diffraction and spatial filtering", Am. J. Phys. Vol. 58, pp. 75-78 (1990).

3. M. E. Knotts, "Scatter-minded: Part II", Optics \& Photonics News, June 1977, pp. 51-52.

4. A. Sommerfeld, "Mathematische Theorie der Diffraction", Math. Ann. Vol. 47, p. 317 (1896).

5. J. W. Goodman, Statistical Optics, J. Wiley \& Sons, New York, 1985, pp. 207-218.

6. P.S. Considine, "Effects of coherence on imaging systems", J. Opt. Soc. Am. Vol. 56, pp. 1001-1009 (1966).

7. M. Born and E. Wolf, Principles of Optics, Pergamon, Oxford, 1975, pp. 428-435; pp. 559-587.

8. E. Hecht and A. Zajac, Optics, Addison-Wesley, Reading, 1974, p. 354.

9. Ref. 7, p. 433 . 